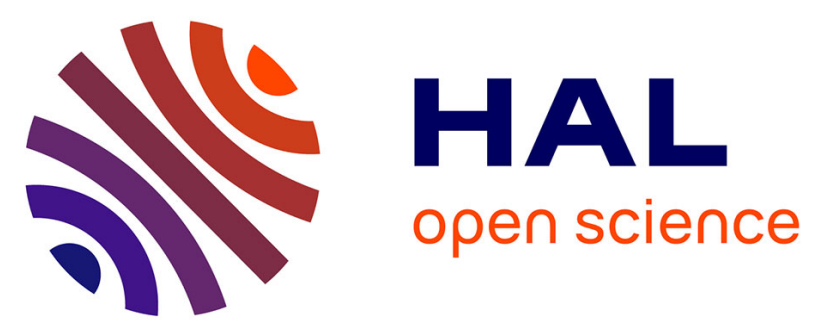

\title{
High frequency characterization of a vertical electro-absorption modulator for data communications
} Ludovic Marigo-Lombart, Christophe Viallon, Alexandre Rumeau, Olivier Gauthier-Lafaye, Antoine Monmayrant, Hugo Thienpont, Krassimir Panajotov, Guilhem Almuneau

\section{To cite this version:}

Ludovic Marigo-Lombart, Christophe Viallon, Alexandre Rumeau, Olivier Gauthier-Lafaye, Antoine Monmayrant, et al.. High frequency characterization of a vertical electro-absorption modulator for data communications. 2018 International Topical Meeting on Microwave Photonics (MWP), IEEE, Oct 2018, Toulouse, France. hal-01980197

\section{HAL Id: hal-01980197 \\ https://hal.laas.fr/hal-01980197}

Submitted on 14 Jan 2019

HAL is a multi-disciplinary open access archive for the deposit and dissemination of scientific research documents, whether they are published or not. The documents may come from teaching and research institutions in France or abroad, or from public or private research centers.
L'archive ouverte pluridisciplinaire HAL, est destinée au dépôt et à la diffusion de documents scientifiques de niveau recherche, publiés ou non, émanant des établissements d'enseignement et de recherche français ou étrangers, des laboratoires publics ou privés. 


\title{
High frequency characterization of a vertical electro-absorption modulator for data communications
}

\author{
L. Marigo-Lombart ${ }^{1,2}$, C. Viallon ${ }^{1}$, A. Rumeau ${ }^{1}$, O. Gauthier-Lafaye ${ }^{1}$, A. Monmayrant ${ }^{1}$, H. Thienpont ${ }^{2}$, \\ K. Panajotov ${ }^{2}$ and G. Almuneau ${ }^{1}$ \\ ${ }^{1}$ LAAS-CNRS, Université de Toulouse, CNRS, Toulouse, France \\ ${ }^{2}$ Department of Applied Physics and Photonics (TW-TONA), Vrije Universiteit Brussel, Pleinlaan 2, \\ B-1050 Brussels, Belgium \\ lmarigol@laas.fr - kpanajot@b-phot.org - almuneau@laas.fr
}

\begin{abstract}
This paper describes the high-frequency characterizations of a vertical electro-absorption modulator (EAM) to ensure its monolithic integration onto a VCSEL for data communications. Splitting the emitting and the modulating part in such devices is an attractive approach compared to directly currentmodulated VCSEL since it releases the intrinsic limitations due to carriers dynamics in such VCSEL device. It is, in this aim, utmost to thoroughly characterize and understand the properties and performances of the vertically integrated EAM under high frequency operation, as it will be decisive for the application of EAM-VCSEL to very high data-rate communications. EAM measurements, presented up to $40 \mathrm{GHz}$, require a precise control of the optical fiber coupling and a precise evaluation of the injection losses over the full frequency range.
\end{abstract}

Index Terms-Modulator, Electro-Absorption, VCSEL, highfrequency

\section{INTRODUCTION}

Nowadays, Vertical-Cavity Surface-Emitting Lasers (VCSELs) raise great awareness for their applications in numerous markets from the optical mouse to the 3D sensing, but primarily to extend the capacity of optical communications within datacenters, with a great concern on power consumption limitation. Indeed, VCSELs bring many advantages compared to edge emitters such as high density integration capabilities and easy coupling in optical fiber, making them ideal as light sources for very high frequency and high capacity optical datalinks. The direct modulation approach has been recently extensively developed and improved through many design strategies, but the physical limitation due to carriers dynamics poses a physical barrier. Therefore it is necessary to move forward to another solution to overcome this fundamental issue. For example, splitting of the emitting/gain and the modulating sections has already been proposed by several groups by combining an electro-optic modulator and a VCSEL, laterally [1] or within a vertical integration scheme. In this last configuration two solutions are possible. The first one is by modulating the refractive index of the top mirror (Distributed Bragg Reflector, DBR), and so the reflective spectrum via an electric field [2]. In that case both cavities are coupled which allows very high bandwidth at the expense of an important chirp and a low transmission data rate. The second one, based on the absorption modulation by quantumconfined Stark effect, in which both optical cavities are weakly coupled, has already reached high frequency bandwidth with regulated temperature [3]. In this project, we focus on this last approach with the vertical integration of an electro-absorption modulator (EAM) onto a CW powered VCSEL. The highfrequency modulation performance relies then only on the modulator itself, independently of the VCSEL light source. The effectiveness of this integrated EAM-VCSEL scheme has already been demonstrated by static measurements with achievable modulation depth of $40 \%$ at a $4 \mathrm{~V}$ reverse bias [4].

In this paper, we focus on the characterization of the high-frequency electro-optic response of the modulator, by measuring its top reflectivity by fiber butt-coupling and using an external laser source. After a short description of the EAM structure and functioning, two measurement setups intended for bandwidth characterization are compared: one with a vector network analyzer (VNA) and the other one with a frequency synthesizer and a spectrum analyzer. For higher measurement accuracy, specially at high frequencies, the implemented InGaAs photodiode response has been measured by heterodyne beat at $850 \mathrm{~nm}$ and $1550 \mathrm{~nm}$ to be compared with the supplier data. Results are finally presented and described while considering all the electrical losses across the injection chain and including the photodiode response.

\section{DEVICES DESCRIPTION}

The EAM is a composed of a multiple quantum wells cavity sandwiched between two DBR to increase the absorption in the active region as described in [4]. The maximum modulation depth is obtained at $838 \mathrm{~nm}$ when a reverse bias, for doped DBR, of $4 \mathrm{~V}$ is applied to modify the band diagram of the structure. A very fast variation of the EAM absorption can be obtained by adding a RF small-signal to the voltage bias, making this EAM a voltage-driven device. Due to the nondoped cavity region (around $500 \mathrm{~nm}$ ) the EAM presents a high 
impedance which drops when the frequency is increased. The schematic of the global EAM-VCSEL device is represented in Fig. 1. We processed the sample for high-frequency operation and thus planarized it with $\mathrm{BCB}$ to decrease the parasitics capacitance and used a microstrip line access for the radiofrequency (RF) signal injection.

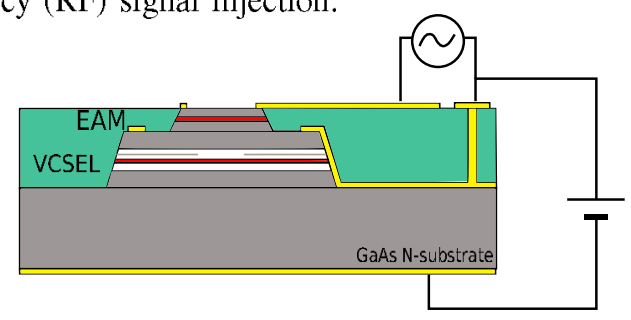

Fig. 1. Schematic of the EAM-VCSEL device powering.

With the characterization setups described below, the proper operation of the modulator is verified for different voltage values and illuminating wavelengths.

\section{DEVICE CHARACTERIZATION}

A dedicated electro-optical characterization setup has been developed to vertically inject and detect light through a singlemode $\mathrm{Y}$ fiber coupler on the modulator top surface. The incident monochromatic light is supplied by a tunable laser source (TLS) and the reflected EAM-modulated signal is measured using a high-speed photodiode as seen in red in Fig. 2. The TLS is a Superlum BS-840-1 (820 - $840 \mathrm{~nm}$, linedwidth $=0.06 \mathrm{~nm}$ ) and the employed InGaAs Discovery DSC20H-39 photodiode displays a cut-off frequency of 32 $\mathrm{GHz}$ at $1550 \mathrm{~nm}$. The RF injection is either carried out by a VNA source or by a distinct RF synthesizer as described in the next subsections. Finally, the $-3 \mathrm{~dB}$ cut-off frequency of the device is obtained by normalizing the photodetected EAM response under RF voltage excitation with the response obtained at the lowest frequency voltage.

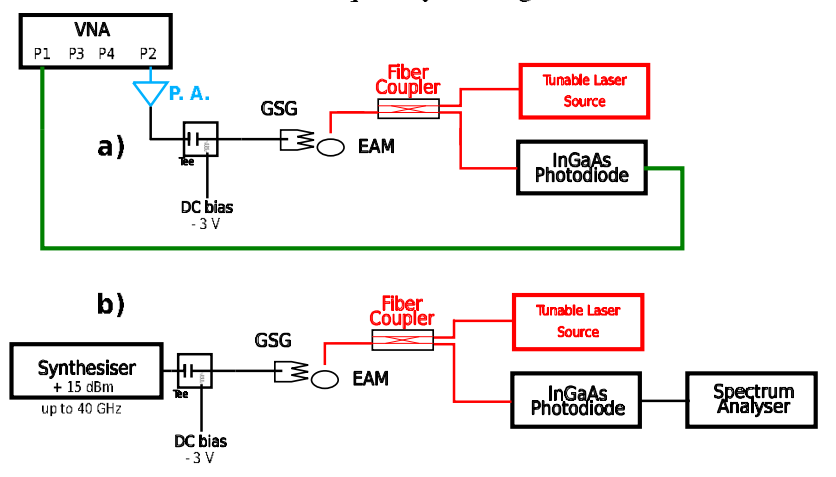

Fig. 2. Schematics of the electro-optic characterization of the EAM with a VNA (a) and with a synthetiser (b).

\section{A. VNA implementation}

The first implementation is displayed in Fig. 2a. A VNA Agilent PNA-X $67 \mathrm{GHz}$ is used to extract the EAM response. Since the signal retrieved by the photodiode is too low due to several cumulative factors (EAM reflectivity, fiber misalignment and photodiode responsivity in the near-infrared range), a power amplifier (PA) featuring a maximum ouptut power of $+24 \mathrm{dBm}$ has been inserted between VNA and EAM RF input. The port 2 is connected to the EAM through this PA, a bias Tee, a coaxial K-band cable and a Cascade Infinity $40 \mathrm{GHz}$ coplanar on-wafer RF probe. Any change in EAM reflectivity is measured by the photodiode and sent to the port 1 . The EAM bandwidth is extracted from the measured transmission parameter $S_{12}$ and the PA-limited $10 \mathrm{MHz}-18 \mathrm{GHz}$ frequency range is covered in one sweep by the VNA.

1) Preliminary results: The first set of measurements is plotted in Fig. 3. As we can see in red, green and black, any modification in the EAM DC voltage bias and TLS wavelength changes the $S_{12}$ magnitude observed on the VNA. This clearly indicates that a modulation effect takes place within the EAM section, thus demonstrating the effective electro-absorption modulation at such frequencies. It is interesting to note that the slope observed between $1 \mathrm{GHz}$ and $18 \mathrm{GHz}$ remains unchanged whatever the voltage and wavelength values.

Jointly, we have measured the electrical losses in the "PA + bias tee + Cable" response, in black at the top in Fig. 3. This result confirms that the slope seen in all $S_{12}$ curves is strongly related to the RF signal injection losses. If these losses are subtracted from the raw transmission measurements, a very flat response from the EAM is obtained all over the $1-18 \mathrm{GHz}$ frequency range (blue curve).

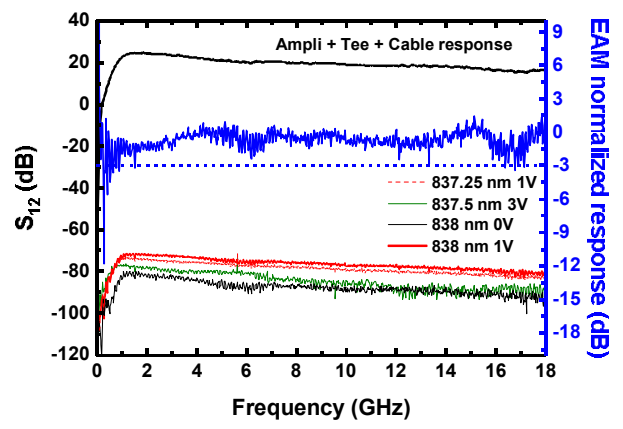

Fig. 3. EAM response $\left(S_{12}\right)$ measured by the VNA-based test setup.

2) Limitations: This result shows that we are especially limited by the bandwidth of the PA and by the losses within the test bench between VNA and EAM device. One solution to overcome these limitations should be to change the available power set at the input of the EAM for each frequency point. Unfortunately such an option is usually not available in VNAs.

\section{B. RF synthesizer and spectrum analyzer}

In this subsection we present the second measurement setup we have developed, which mainly consists in replacing the VNA by an RF synthesizer (Anritsu MG 3694B) coupled with a spectrum analyzer (Fieldfox N9951 A from Keysight) at the photodiode output. This second version of the measurement bench is shown in Fig. 2b. The most important improvements brought by this new configuration are the nearly constant power delivered by this RF synthesizer all over the $40 \mathrm{GHz}$ bandwidth as well as the pre-amplifier integrated within the spectrum analyzer which increases the electrical signal level issued by the photodiode. 
1) Calibration: We have gained a valuable experience with previous measurements and we now know that the losses calibration is the key point of the setup. Firstly, the output power of the synthesizer, set to a value of $+15 \mathrm{dBm}$, is verified by using a powermeter (Anritsu ML 2437A). This measurement value, plotted in Fig. 4 (black curve), presents a delta of $0.9 \mathrm{~dB}$ in the $1-40 \mathrm{GHz}$ frequency range. The insertion of the bias tee and cable system lowers the available power by a maximum value of $3.5 \mathrm{~dB}$ in the same frequency range (blue curve). These losses have to be compensated to extract a more realistic EAM response.

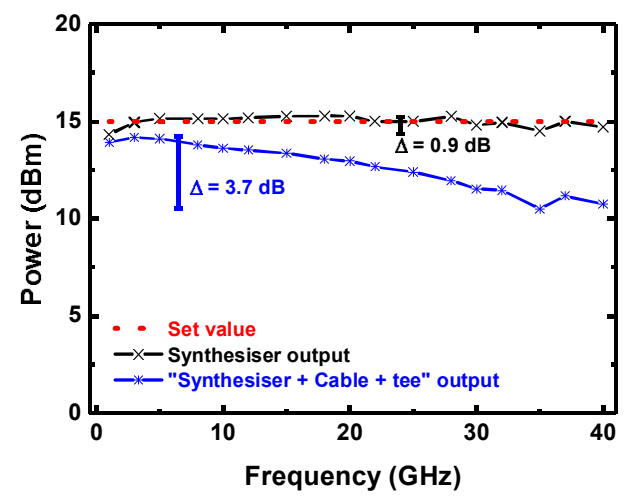

Fig. 4. Power calibration of the RF synthetiser set to $+15 \mathrm{dBm}$ alone (black curve) and with the system "bias tee + cable" (blue curve).

2) Preliminary results: Previous results are used to adjust the power delivered by the RF synthesizer so as to provide a constant value of $+15 \mathrm{dBm}$ at the input of the RF probe connected to EAM pads. Several measurements carried on various EAM (diameters from 18 to $27 \mu \mathrm{m}$ ) integrated alones or onto VCSELs have revealed that the VCSEL cavity has no influence on EAM response. But whatever the tested configuration, a constant $-5 \mathrm{~dB} / \mathrm{dec}$ slope is still observed all over the measured frequency range. However, since the cutoff frequency is linked to the capacitance of the device and thus to its mesa diameter, we should have observed different slopes for each tested diameter. The absence of difference clearly indicates that this observed slope cannot be traced to the low-pass nature of EAM devices. RF probes, EAM pad and microstrip access line contribution to the overall insertion losses have been neglected since it does not exceed $1 \mathrm{~dB}$ at $40 \mathrm{GHz}$ it cannot explain this $-5 \mathrm{~dB} / \mathrm{dec}$ slope too.

3) Losses compensation: As already stated before, the EAM RF input presents a very high impedance. All the measurement chain starting from RF source up to the RF probe tips is loaded by an impedance approaching an open circuit at the lowest frequencies. This impedance decreases with increasing frequencies but still remains much larger than $50 \Omega$ at $40 \mathrm{GHz}$. The determination of the EAM response based on the measurement of the available power cannot be satisfactory due to this frequency varying mismatch. Since the EAM is fundamentally a voltage driven device, a more natural approach is to use the applied voltage magnitude to recover its frequency response. This voltage can be assessed from the knowledge of the available power from RF source, the scattering parameters of the test setup, and the EAM impedance. This problem is illustrated in Fig.5. The RF source is modeled by its Thevenin generator $V_{q}$ followed by a serie resistance $R_{q}=50 \Omega=R_{0}$. The test setup comprising the bias tee, the cable and the RF probe is depicted by the two-port network and its scattering matrix. Finally, $V_{L}$ is the voltage across the input impedance of the EAM.

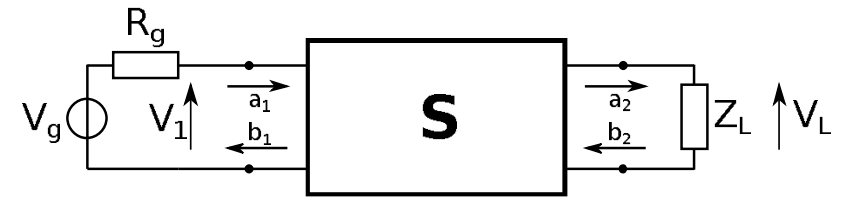

Fig. 5. Schematic of the RF signal injection from RF source to EAM input.

Let $\Gamma_{L}, \Gamma_{g}$ and $\Gamma_{i n}$ be the reflection coefficients displayed by the EAM, the RF source and the two-port network input, respectively.

$$
\begin{gathered}
\Gamma_{L}=\frac{Z_{L}-R_{0}}{Z_{L}+R_{0}} \\
\Gamma_{g}=\frac{R_{g}-R_{0}}{R_{g}+R_{0}} \\
\Gamma_{i n}=S_{11}+\frac{S_{12} S_{21} \Gamma_{L}}{1-S_{22} \Gamma_{L}}
\end{gathered}
$$

The signal flow graph technique [6] is used to find the relationship between voltage $V_{L}$ and $V_{g}$, using the two-port scattering parameters, $\Gamma_{L}, \Gamma_{g}$ and $\Gamma_{i n}$ :

$$
V_{L}=\frac{S_{21}}{2} \cdot \frac{1+\Gamma_{L}}{1-\Gamma_{L} S_{22}} \cdot \frac{1-\Gamma_{g}}{1-\Gamma_{g} \Gamma_{i n}} \cdot V_{g}
$$

$V_{g}$ is deduced from the available power measured across $R_{0}$ which is also the input impedance of the powermeter:

$$
P_{\text {disp }}=\frac{1}{2} \cdot \frac{\left|V_{g}\right|^{2}}{4 R_{0}} \Longrightarrow\left|V_{g}\right|=\sqrt{8 R_{0} P_{\text {disp }}}
$$

In practice, the complete two-port scattering matrix cannot be measured easily because of the different nature of input (SMA cable) and output (RF probe tips) terminals of this box. However, the matrix is symmetric and $S_{12}=S_{21}$. $S_{i j}$ parameters are extracted from measurements carried out using a SHORT or OPEN circuit at RF probe ends while $S_{11}=S_{22}$ are assumed to be zero, which is not too far from reality. Using this assumption, $\left|V_{L}\right|$ becomes

$$
\left|V_{L}\right|=\left|S_{21}\right| \cdot\left|1+\Gamma_{L}\right| \cdot \sqrt{2 R_{0} P_{\text {disp }}}
$$

With this last equation, we are now able to link the EAM voltage to the available power measured at the RF source output. The idea is now to calculate the power we need to set so as to maintain a constant voltage at EAM pads all over the studied frequency range.

\section{Photodiode characterization and results}

The last thing to validate in the electro-optical setup is the photodiode frequency response at $850 \mathrm{~nm}$. In previous measurements we dealt with the supplier data given at 1550 $\mathrm{nm}$ but the response may be different at $850 \mathrm{~nm}$. Hence, the photodiode response has been measured by using an 
heterodyne beating technique at $850 \mathrm{~nm}$ and and also at 1550 $\mathrm{nm}$ to compare the results with the supplier data.

At $1550 \mathrm{~nm}$ we used an InGaAs DFB at $1537.2 \mathrm{~nm}$ regulated by an IXL Ligthwave LDC-3722 B at $22.9{ }^{\circ} \mathrm{C}$ biased at $70.1 \mathrm{~mA}$ to deliver $-3 \mathrm{dBm}$ in the photodiode under test. The used tunable laser is a Santec TSL-510 and is conveyed to the photodiode through a polarization maintaining coupler. At $850 \mathrm{~nm}$, the stationary laser is a homemade laser formed with a LED and a Cavity Resonator Integrated Grating Filter (CRIGF) [5] which filters a precise wavelength, here $854 \mathrm{~nm}$. The LED is biased at $52 \mathrm{~mA}, 1.7 \mathrm{~V}$ and is maintained at $20^{\circ} \mathrm{C}$ to maintain single mode operation. The other laser is a Vantage TLB7100 from Newfocus. For both measurements, the photodiode response is sent in a Rohde \& Schwarz spectrum analyzer and is extracted from $20 \mathrm{~Hz}$ up to $40 \mathrm{GHz}$. For each wavelength we acquired 10 spectra and did an average to decrease the uncertainty due to the vibration or air flow in the room.

The results are presented in Fig. 6. At $1550 \mathrm{~nm}$, the same response than supplier data is obtained which makes us confident with the result displayed at $850 \mathrm{~nm}$. As we can see, the photodiode response at $850 \mathrm{~nm}$ is really different from the one at $1550 \mathrm{~nm}$. This new characteristic is used to improve the measurement precision of the modulator cut-off frequency.

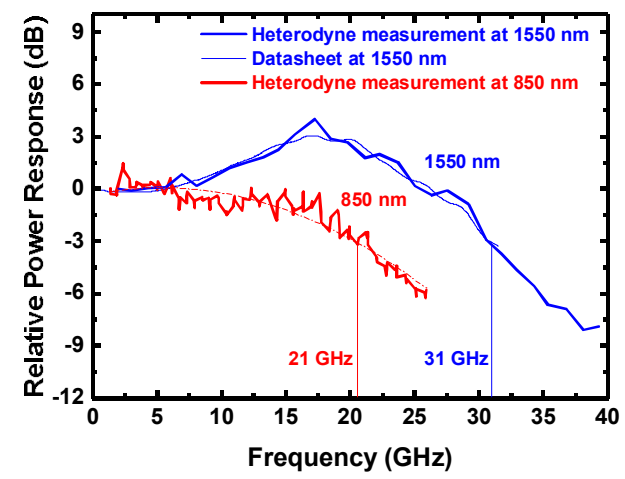

Fig. 6. Chracterization of the $\mathrm{InGaAs}$ photodiode at $1550 \mathrm{~nm}$ (blue) and 850 nm (red).

We then measured a $27 \mu$ m-diameter EAM at $838 \mathrm{~nm}$ with a bias offset of $3 \mathrm{~V}$ and a RF signal amplitude of $0.8 \mathrm{~V}$ at the EAM pad plane. The signal detected by the InGaAs photodiode is presented in Fig. 7. The effects of the photodiode responses compensation are displayed. As shown in Fig. 7, the cut-off frequency of the EAM is evaluated up to more than $23 \mathrm{GHz}$, instead of around $15 \mathrm{GHz}$ when considering the photodiode supplier characteristics at $1550 \mathrm{~nm}$. The EAM high frequency response could not be further characterized above $23 \mathrm{GHz}$, since the photodiode responsivity drops drastically.

\section{CONCLUSION}

In this paper we present different setups for the electrooptical characterization of a vertical EAM. First, with a VNA but this characterization turns out to be limited by the frequency range of the power amplifier used to measure the modulated signal. And secondly, we proposed an original setup

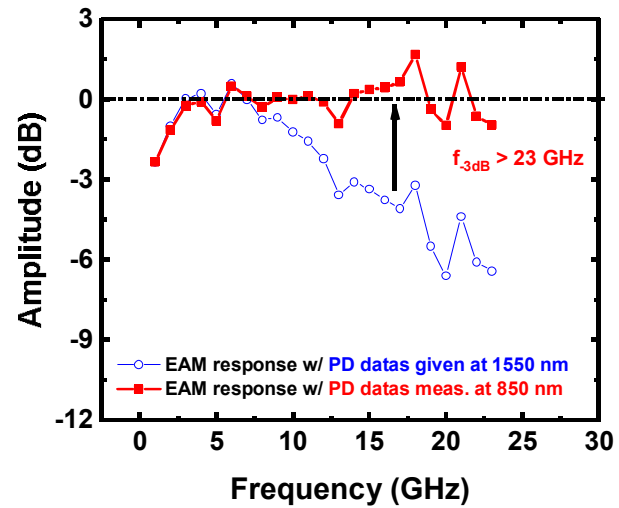

Fig. 7. High frequency characterization of the $27 \mu \mathrm{m}-\mathrm{EAM}$.

involving a RF synthesizer along with a spectrum analyzer to extend the measurement frequency range up to $40 \mathrm{GHz}$. This setup finally appears limited by the responsivity of the photodiode. Thanks to these improvements, we are able to measure a cut-off frequency above $23 \mathrm{GHz}$ for this vertical GaAs-based asymmetric Fabry-Perot electro-absorption modulator. This result can be compared to the $20 \mathrm{GHz}$ modulation demonstrated in [3] which employs a similar approach. To our knowledge, this result is the best achieved modulation bandwidth for this type of architecture, compatible with the monolithic integration onto a VCSEL source.

\section{ACKNOWLEDGMENT}

The authors gratefuly acknowledge the technological support of RENATECH (French Network of Technology Platforms) within LAAS-CNRS cleanroom infrastructure and the financial support by the Methusalem foundation, Belgium.

\section{REFERENCES}

[1] X. Gu, M. Nakahama, A. Matsutani, M. Ahmed, A. Bakry and F. Koyama, "850 nm transverse-coupled-cavity vertical-cavity surfaceemitting laser with direct modulation bandwidth of over $30 \mathrm{GHz}$ ", Appl. Phys. Expr. 8, 082702, 2015

[2] T. D. Germann, A. Strittmatter, A. Mutig, A. M. Nadtochiv, J. A. Lott, S. A. Blokhin, et al., "Monolithic electro-optically modulated vertical cavity surce emitting laser with $10 \mathrm{~Gb} / \mathrm{s}$ open-eye operation", Phys. Status Solidi C 7, No. 10, 2552-2554, 2010

[3] J. van Eisden, M. Yakimov, V. Tokranov, M.Varanasi, O. Rumyantsev, E. M. Mohammed, et al. "High Frequency Resonance-Free Loss Modulation in a Duo-Cavity VCSEL", Proc. of SPIE Vol. 6908, 69080M, 2008

[4] L. Marigo-Lombart, S. Calvez, A. Arnoult, H. Thienpont, G. Almuneau and K. Panajotov, "Vertical electro-absorption modulator design and its integration in a VCSEL", J. Phys. D: Appl. Phys. 51, 2018

[5] X. Buet, A. Guelmami, A. Monmayrant, S. Calvez, C. Tourte, F. Lozes-Dupuy, and O. Gauthier-Lafaye, "Wavelength-stabilised externalcavity laser diode using cavity resonator integrated guided mode filter", Electronics Letters, 48(25), 1619-1621, 2012

[6] D. M. Pozar, Microwave Engineering, 4th ed. New York, NY, USA: Wiley, 2011. 